nicht invariant ist. [Man kann annehmen, daß die Invarianz (7) zur Erhaltung der Baryonenzahl führt.]

Aus (3) sieht man unmittelbar, daß $L$ nur dann hermitesch ist, wenn $\lambda_{1221}=\lambda_{2112}$ ist. Gegen Drehungen und Spiegelungen im Isotopenraum invariante LAGRANGEFunktionen gewinnen wir bei folgenden Koeffizienten

$\lambda_{1221}=\lambda_{2112}=0, \lambda_{1111}=\lambda_{1122}=\lambda_{2211}=\lambda_{2222}$,

$\lambda_{1221}=\lambda_{2112}=2 \lambda_{1111}, \lambda_{1111}=-\lambda_{1122}=-\lambda_{2211}=\lambda_{2222}$,

$\lambda_{1122}=\lambda_{2211}=2 \lambda_{1111}, \lambda_{1111}=-\lambda_{1221}=-\lambda_{2112}=\lambda_{2222}$.

Es werden nun die Folgen der Wahl $\lambda_{2222}=0$ untersucht. Das bedeutet anschaulicherweise, daß die in der Näherung $n=2$ durch die Amplitude

$$
\left\langle\Omega\left|T \psi_{2}(x) \psi_{2}^{+}(y)\right| \Phi\right\rangle
$$

beschriebenen $\gamma$-Bosonen nur zwischen den durch den Operator $\psi_{1}$ erzeugten Fermionen eine Wechselwirkung hervorrufen. Wenn es im Falle des $\gamma$-Bosons eine Lösung gibt, die einem vektoriellen Boson mit einer Masse gleich Null oder annähernd Null entspricht, so kann diese mit dem Photon gleichgesetzt und dementsprechend $\psi_{1}$ als geladenes, $\psi_{2}$ als neutrales Feld angesehen werden. Nun ist zu erwarten, daß sich die elektrische Ladung aus der Theorie unabhängig vom Teilchenzustand immer gleich ergibt. Die Annahme ist naheliegend, daß die $\pi$-Mesonen in der Näherung $n=2$ durch folgende Amplituden beschrieben sind

$$
\begin{aligned}
& \pi^{+} \rightarrow\left\langle\Omega\left|T \psi_{1}(x) \psi_{2}{ }^{+}(y)\right| \Phi\right\rangle, \\
& \pi^{0} \rightarrow\left\langle\Omega\left|T \psi_{1}(x) \psi_{1}{ }^{+}(y)\right| \Phi\right\rangle, \\
& \pi^{-} \rightarrow\left\langle\Omega\left|T \psi_{2}(x) \psi_{1}{ }^{+}(y)\right| \Phi\right\rangle .
\end{aligned}
$$

Die Analogie mit der Theorie von de Broglie ${ }^{5}$ und mit den Annahmen von FERMI und $\mathrm{Y}_{\mathrm{ANG}}{ }^{6}$ ist hervorzuheben. Unsere Grundannahme ist, daß infolge der

5 L. de Broglie. Théorie Generales des Particules a Spin, Gauthier-Villars, Paris 1954.

6 E. FerMI u. N. C. YANG, Phys. Rev. 76, 1739 [1949].
Verletzung der Isoinvarianz annähernd stationäre $\gamma$ und $\pi^{0}$-Amplituden existieren.

Mit Berücksichtigung der Obigen können die Werte von drei Koeffizienten festgesetzt werden.

$$
\lambda_{1221}=\lambda_{2112}=-\lambda_{1111}=\lambda_{2222}=0 .
$$

Aus der Untersuchung der Feldgleichungen ist nämlich zu ersehen, daß die Kopplungskonstante der Protonen bzw. Neutronen und des $\pi^{0}$-Feldes aus der Theorie bei der Wahl (16) sich richtig ergeben kann. (Wir nehmen an, daß die $\gamma$-Bosonen hauptsächlich im Photonenzustand entstehen.) Die Werte der Konstanten $\lambda_{1122}$ und $\lambda_{2211}$ müssen so gewählt werden, daß die Ladungsunabhängigkeit der nuklearen Wechselwirkungen gesichert ist. (Die nähere Untersuchung der Annahmen

$$
\begin{array}{ll}
\lambda_{1122}=2 \sqrt{2} \lambda_{1111}, & \lambda_{2211}=0, \\
\lambda_{2211}=2 \sqrt{2} \lambda_{1111}, & \lambda_{1122}=0
\end{array}
$$

scheint zweckmäßig zu sein.) Mit Berücksichtigung der Tatsache, daß die Massen der $\pi$-Mesonen annähernd gleich sind, ist zu erwarten, daß zur Bindung des $\pi^{0}$. Mesons nur das Glied mit dem Koeffizienten $\lambda_{1111}$ einen wesentlichen Beitrag liefert.

Im Zusammenhang mit dem Problem der Quantisierung weisen wir auf die früheren Arbeiten von HeisenBERG hin.

Zum Schluß nehmen wir an, daß die $x$-Mesonen in einem „asymmetrischen" Zustand sind und ihre Wechselwirkung den Nukleonen ähnlich sind. Als Modell kann man ein 2-Fermionensystem erwähnen, wo nur das eine Fermion ein stark wechselwirkendes Teilchen ist. Wenn so ein „asymmetrischer" Zustand existiert, dann bringt die Behandlung der schweren instabilen Teilchen und ihrer Wechselwirkungen - in analoger Weise mit dem Modell von Goldhaber ${ }^{7}$ und GyörgYI ${ }^{8}$ - voraussichtlich keine prinzipiellen Schwierigkeiten.

7 M. Goldhaber, Phys. Rev. 101, 433 [1956].

8 G. Györqyi, J. Exp. Theor. Phys., USSR 32, 152 [1957]. Soviet Phys. J. Exp. Theor. Phys. 5, 152 [1957].

\section{Energie der Neutronen vom Einfang negativer $\mu$-Mesonen in Bleikerne}

Von W. Ball und K. H. Lauteruung

Max-Planck-Institut für Kernphysik, Heidelberg

(Z. Naturforschg. 14 a, 581-582 [1959]; eingegangen am 24. April 1959)

Beim Einfang negativer $\mu$-Mesonen in schweren Kernen werden bevorzugt Neutronen emittiert ${ }^{1}$. In dem hier beschriebenen Versuch wurden die bei den Einfangprozessen in Blei entstehenden Neutronen durch die von ihnen in Paraffin ausgelösten Rückstoßprotonen in $\mathrm{ZnS}(\mathrm{Ag})$ Leuchtschirmen nachgewiesen. Die Absorptionsmessungen an den Rückstoßprotonen erlauben dann Aussagen über die Energie dieser Neutronen. Abb. 1 zeigt die benutzte Versuchsanordnung. Die Zählrohre der Lagen A und B waren in Koinzidenz geschaltet und wählten diejenigen geladenen Teilchen der Höhenstrahlung aus, die zwischen $\mathrm{A}$ und $\mathrm{B}$ durch die Bleilage $\mathrm{Pb}$ von insgesamt $165 \mathrm{~g} / \mathrm{cm}^{2}$ Luftäquivalent hindurchgegangen waren. Die damit gemessene Koinzidenzzählrate AB besteht zu 99\% aus positiven und negativen $\mu$-Mesonen ${ }^{2}$. Die Zählrohrlagen $\mathrm{A}$ und $\mathrm{B}$ waren mit den Zählrohren der Gruppen $\mathrm{C}_{0}-\mathrm{C}_{4}$ in Antikoinzidenz geschaltet, so daß von der Antikoinzidenzstufe $\mathrm{AB}-\mathrm{C}$ nur dann ein Impuls weitergegeben wurde, wenn Zählrohre der Lagen A und B gleichzeitig angesprochen hatten, nicht aber Zählrohre der Gruppen $\mathrm{C}_{0}-\mathrm{C}_{4}$. Mit der so definierten Antikoinzidenzzählrate $\mathrm{AB}-\mathrm{C}$ wurde aus der Differenz der

1 R. D. Sard u. M. F. Crouch, Prog. Cosmic Ray Phys. 2, 3 [1954] ; L. Winsberg, Phys. Rev. 95, 205 [1954].

2 B. Rossi, Rev. Mod. Phys. 20, 537 [1948]. 
Messungen mit und ohne Bleiabsorber $\mathrm{D}$ die Zahl der in der Zeiteinheit im Absorber abgestoppten $\mu$-Mesonen bestimmt. Die beim Einfang der negativen $\mu$-Mesonen entstehenden Neutronen wurden durch Rückstoßprotonen mit den Detektoren ${ }^{3} \mathrm{~S}_{1}-\mathrm{S}_{4}$ nachgewiesen, die in Koinzidenz mit der Antikoinzidenzstufe $\mathrm{AB}-\mathrm{C}$ geschaltet waren. Ein $(\mathrm{AB}-\mathrm{C})$ N-Koinzidenzimpuls repräsentiert demnach ein Zählereignis, bei dem in den Bleiabsorber D ein $\mu$-Meson ein- aber nicht ausgetreten ist, und ein oder mehrere Zähler des Neutronendetektors innerhalb der Koinzidenzauflösezeit angesprochen haben.

Die Protonen wurden durch Al-Folien ${ }^{4}$ absorbiert, die zwischen Paraffin und Leuchtschirm der Neutronendetektoren eingeschoben waren. In der Tabelle sind die für verschiedene Al-Absorberdicken registrierten Rückstoßprotonen-Zählraten mit dem einfachen statistischen Fehler aufgeführt.

\begin{tabular}{|c|c|c|c|}
\hline 1 & 2 & 3 & 4 \\
$\begin{array}{c}\mu \text {-Mesonen- } \\
\text { absorber }\end{array}$ & $\begin{array}{c}\text { Al-Absorber } \\
\text { in } \\
\mathrm{mg} / \mathrm{cm}^{2}\end{array}$ & $\begin{array}{c}\text { Energie der } \\
\text { Rückstoß- } \\
\text { protonen in } \\
\text { MeV }\end{array}$ & $\begin{array}{c}\text { Gemessene Rück- } \\
\text { stoßprotonen- } \\
\text { zählrate pro } \\
\text { Stunde }\end{array}$ \\
\hline & & & $0,045 \pm 0,033$ \\
$\mathrm{~Pb}$ & 4,05 & $>1,7$ & $0,041 \pm 0,030$ \\
& & & $0,046 \pm 0,013$ \\
$\mathrm{~Pb}$ & 4,05 & $>1,7$ & $0,045 \pm 0,011$ \\
$\mathrm{~Pb}$ & 5,40 & $>1,9$ & $0,029 \pm 0,013$ \\
$\mathrm{~Pb}$ & 6,75 & $>2,1$ & $0,022 \pm 0,014$ \\
$\mathrm{~Pb}$ & 8,10 & $>2,3$ & $0,006 \pm 0,012$ \\
\hline
\end{tabular}

Die Tabelle zeigt, daß der größte Teil aller nach dem Einfang negativer $\mu$-Mesonen in Blei emittierten Neu-

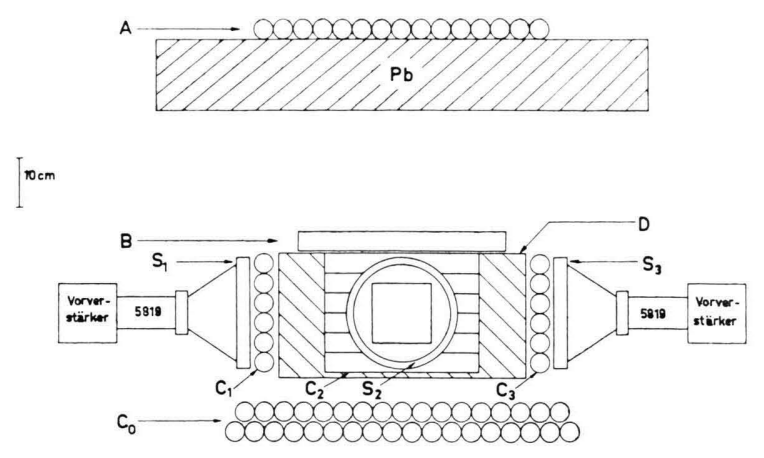

Abb. 1. Versuchsanordnung. A, B, $\mathrm{C}_{0}, \mathrm{C}_{1}, \mathrm{C}_{2}, \mathrm{C}_{3}$ (und $\mathrm{C}_{4}$ verdeckt) Zählrohrgruppen des Höhenstrahlenteleskopes, $S_{1}$, $\mathrm{S}_{2}, \mathrm{~S}_{3}$ (und $\mathrm{S}_{4}$ verdeckt) Szintillationszähler des Neutronendetektors, D $\mu$-Mesonenabsorber.

tronen Energien kleiner als $3 \mathrm{MeV}$ hat ${ }^{5,6}$. Da Neutronen die entsprechend ihrem Entstehungsort große Wegstrecken im $\mu$-Mesonenabsorber zu durchlaufen haben, auf Grund ihres Energieverlustes durch unelastische Stöße den Bleiabsorber als niederenergetische Neutronen verlassen, ist die Gesamtintensität der Rückstoßprotonen, obwohl im Mittel 25 Einfangprozesse/min registriert wurden, sehr klein. Die Rückstoßprotonenzählrate würde jedoch größer sein, wenn bevorzugt Neutronen mit Energien größer als $25 \mathrm{MeV}$ zu erwarten wären ${ }^{7}$.

3 W. Ball u. K. H. Lauterjung, im Druck.

${ }^{4}$ E. Segrè, Experimental Nuclear Physics, Vol. I, New York, London 1952, p. 219.

5 M. Widgoff, Phys. Rev. 90, 891 [1953].

${ }^{6}$ C. T. Coffin, A. M. Sachs u. D. H. Tycko, Bull. Amer. Phys. Soc. II, 3, 52 [1958].

7 L. Niklas u. K. H. Lauterjung, Z. Naturforschg. 8 a, 214 [1953].

\section{Diffusion von Xe-133 in Uranoxyd verschiedenen Sauerstoffgehaltes}

\author{
Von R. Lindner und HJ. Matzke \\ Kernchemisches Institut * der Technischen Hochschule, \\ Göteborg, Schweden \\ (Z. Naturforschg. 14 a, 582-584 [1959]; eingegangen am 24. April 1959)
}

Nachdem in einer vorangegangenen Mitteilung ${ }^{1}$ die grundsätzliche Möglichkeit der Messung der Diffusion radioaktiven Xenons in Uranoxyden bei verhältnismäßig tiefen Temperaturen gezeigt wurde, sollen hier genauere Werte für die Diffusion in Urandioxyd verschiedenen Sauerstoffgehaltes (sowie in $\mathrm{U}_{3} \mathrm{O}_{8}$ ) bei verschiedenen Temperaturen gegeben werden.

$\mathrm{Zu}$ diesen Versuchen wurde eine neuaufgestellte Apparatur verwendet (vgl. Abb. 1). Mit ihr ist die kontinuierliche Messung des aus der Diffusionsprobe entweichenden Xenons möglich mit Hilfe eines Zirkula- tionssystemes, wobei Argon (mitunter mit Wasserstoffzusatz) als Trägergas benutzt wurde.

Die Diffusionsproben (etwa 5-20 mg im Reaktor mit $\sim 10^{17} \mathrm{n} \cdot \mathrm{cm}^{-2}$ in argongefüllten Quarzampullen bestrahlten Uranoxydes bekannter Korngröße) wurden in einem Quarzschiffchen in das Reaktionsgefäß (1) eingeführt, und dieses mittels Schliff an die Apparatur angeschlossen. Mit einem konventionellen Pumpstand mit zugehörigen Vakumetern (6) wurde die gesamte Apparatur evakuiert $\left(10^{-5}\right.$ Torr $)$ und mehrfach mit gereinigtem Argon gespült, Der verbleibende Argondruck in der Apparatur wurde so gewählt, daß bei Versuchstemperatur ein Überdruck von $10-20$ Torr vorlag. Die Temperatur der Versuchsprobe wurde durch ein $\mathrm{Pt} / \mathrm{Pt}$. Rh-Thermoelement in unmittelbarem Kontakt mit dem Quarzschiffchen gemessen; die Temperatur des überschiebbaren Ofens (13) wurde mit einem zweiten

* Betrieben vom Schwedischen Atomkomitee.

1 R. Lindner u. HJ. Matzke, Z. Naturforschg. 13 a, 294 [1958]. 